\title{
Development and natural course of lateral posterior choroidal artery aneurysms arising from fragile choroidal collaterals in moyamoya disease: illustrative cases
}

\author{
Tomoaki Suzuki, MD, PhD, ${ }^{1}$ Hitoshi Hasegawa, MD, PhD, ${ }^{1}$ Kouichirou Okamoto, MD, PhD, ${ }^{2}$ Kazuhiro Ando, MD, ${ }^{1}$ Kohei Shibuya, MD, \\ Haruhiko Takahashi, MD, ${ }^{1}$ Shoji Saito, MD, ${ }^{1}$ Makoto Oishi, MD, PhD, ${ }^{1}$ and Yukihiko Fujii, MD, PhD ${ }^{1}$
}

Departments of ${ }^{1}$ Neurosurgery and ${ }^{2}$ Translational Research, Brain Research Institute, Niigata University, Niigata, Japan

BACKGROUND Choroidal collaterals are a risk factor for hemorrhagic stroke, even in the nonhemorrhagic hemisphere, among patients with moyamoya disease (MMD). Peripheral choroidal aneurysms rupture in fragile collaterals; however, the development and natural course of these aneurysms remain elusive.

OBSERVATIONS A 51-year-old woman, who had experienced a right cerebral hemorrhage 3 years earlier, presented with asymptomatic minor bleeding from a left lateral choroidal artery aneurysm in a predeveloped choroidal anastomosis. Although the aneurysm spontaneously thrombosed within 2 months, the choroidal collaterals persisted. After bypass surgery, the choroidal anastomosis regressed, and neither a de novo aneurysm nor a hemorrhagic stroke occurred. A 75-year-old woman with MMD, who had experienced a left frontal infarction 6 years earlier, experienced recurrent right intraventricular hemorrhage from a ruptured lateral choroidal artery aneurysm that developed in the choroidal anastomosis. The aneurysm spontaneously regressed 3 days after the rebleeding with no recurrence over the following 7 years.

LESSONS Choroidal artery aneurysms may develop in the choroidal anastomosis and rupture in the nonsurgical or contralateral hemispheres. Patients with MMD who have a history of hemorrhagic or ischemic stroke and impaired cerebral blood flow require careful observation. Although aneurysms may rapidly regress spontaneously, bypass surgery can stabilize hemodynamic stress and prevent further hemorrhage.

https://thejns.org/doi/abs/10.3171/CASE2110

KEYWORDS moyamoya disease; hemorrhage; choroidal artery aneurysm; choroidal anastomosis

Associated hemorrhagic events in moyamoya disease (MMD) have high morbidity and mortality rates. ${ }^{1,2}$ Recently, the Japan Adult Moyamoya (JAM) Trial, a multicenter randomized controlled trial on hemorrhagic MMD, revealed that periventricular anastomoses are dangerous collateral vessels associated with the potential risk of rebleeding in patients with hemorrhagic MMD. ${ }^{3,4}$ In fragile collateral anastomoses, the posterior type, which includes choroidal anastomoses, needs to be carefully managed because of the higher risk of rebleeding. ${ }^{5,6}$ Meanwhile, the subanalysis of the JAM Trial demonstrated that the risk of hemorrhage in the nonhemorrhagic hemisphere with choroidal anastomosis is higher than that without choroidal anastomosis, and it resembles the risk of hemorrhage in asymptomatic patients with MMD. ${ }^{7}$ As the origin of fatal hemorrhage, the formation of MMD-associated intracranial aneurysms has been reported, and these aneurysms are observed in 3-14\% of the patients. ${ }^{8}$ The aneurysms are classified as either major artery aneurysms or peripheral aneurysms. Peripheral aneurysms are characterized by MMD caused by distal dilated fragile collateral arteries exposed to unstable hemodynamic stress. ${ }^{9-12}$ Distal choroidal artery aneurysms in the periventricular region with the risk of hemorrhage in the presence of such fragile collateral vessels in the periventricular region have been described. Although major artery aneurysms can be treated surgically, surgical intervention for peripheral aneurysms is challenging because of their peripheral and deep location. The development and natural history of peripheral choroidal artery aneurysms arising from choroidal collaterals have not been fully elucidated. Herein, we report on 2 cases of newly developed choroidal artery aneurysms in the nonsurgical and nonhemorrhagic hemisphere of 2 adult

\footnotetext{
ABBREVIATIONS CT = computed tomography; CTA = computed tomography angiography; CVR = cerebrovascular reactivity; DSA = digital subtraction angiography; IVH = intraventricular hemorrhage; JAM = Japan Adult Moyamoya; MMD = moyamoya disease; MRA = magnetic resonance angiography; MRI = magnetic resonance imaging; TIA $=$ transient ischemic attack.
}

INCLUDE WHEN CITING Published online April 12, 2021; DOI: 10.3171/CASE2110.

SUBMITTED January 30, 2021. ACCEPTED February 3, 2021.

(C) 2021 The authors, CC BY-NC-ND 4.0 (http://creativecommons.org/licenses/by-nc-nd/4.0/). 
patients with MMD who had previous hemorrhagic and ischemic stroke events.

\section{Illustrative Cases}

\section{Case 1}

A 51-year-old woman presented with asymptomatic minor bleeding from a small left peripheral periventricular aneurysm in the posterior choroidal anastomosis with perifocal edema, detected using magnetic resonance imaging (MRI) at a regular outpatient follow-up (Fig. 1). She had a history of intracranial hemorrhage in the right cerebral hemisphere and intraventricular hemorrhage (IVH) 3 years prior to this episode of care (Fig. 2A). She had no other medical or family history. At that time, examination revealed MMD (Suzuki stage III bilaterally) with a well-developed abnormal anastomosis of the medullary artery in the periventricular region in the right hemisphere (Fig. 2B). A choroidal anastomosis in the nonhemorrhagic left cerebral hemisphere was observed on magnetic resonance angiography (MRA) and digital subtraction angiography (DSA) imaging without the aneurysm (Fig. 2C-E). Single-photon emission computed tomography (CT) with $\mathrm{N}$-isopropyl $\left[{ }^{123} \mathrm{l}\right]$-p-iodoamphetamine evidenced the impaired cerebrovascular reactivity (CVR) in the left hemisphere (Fig. 2F). Bypass surgery was performed for the right hemisphere to prevent rebleeding (17 months after hemorrhage). No surgical intervention was performed for the left hemisphere while she was hospitalized because it was asymptomatic.
Cerebral angiography was planned for further investigation of the left periventricular aneurysm soon after aneurysm detection; however, the procedure was performed 52 days after the aneurysm was detected owing to the patient's personal reasons. The DSA demonstrated no obvious aneurysm of the left posterior choroidal artery and a developed posterior choroidal anastomosis in the left cerebral hemisphere (Fig. 3A). The result of MRI corroborated the DSA finding, and spontaneous regression of the aneurysm was suspected (Fig. 3B-E). Although the aneurysm was thrombosed, the choroidal anastomosis with connection of the medullary artery persisted (Fig. 3F). Five months after the asymptomatic minor bleeding event, bypass surgery (superficial temporal artery-middle cerebral artery anastomosis and encephaloduromyosynangiosis) was performed to prevent recurrence of the aneurysm and hemorrhagic stroke of the left cerebral hemisphere. On follow-up MRA 2 weeks after surgery, bypass patency was confirmed and shrinkage of the choroidal anastomosis was already observed. There was no recurrence of the aneurysm (Fig. $3 G$ and $H$ ).

\section{Case 2}

A 75-year-old woman, previously diagnosed with MMD (Suzuki stage III bilaterally) with a left frontal infarction and dilated right posterior choroidal artery with choroidal-type periventricular anastomosis (Fig. $4 \mathrm{~A}-\mathrm{C}$ ), presented with a complaint of sudden-onset severe headache and was admitted to the hospital. A CT scan revealed a
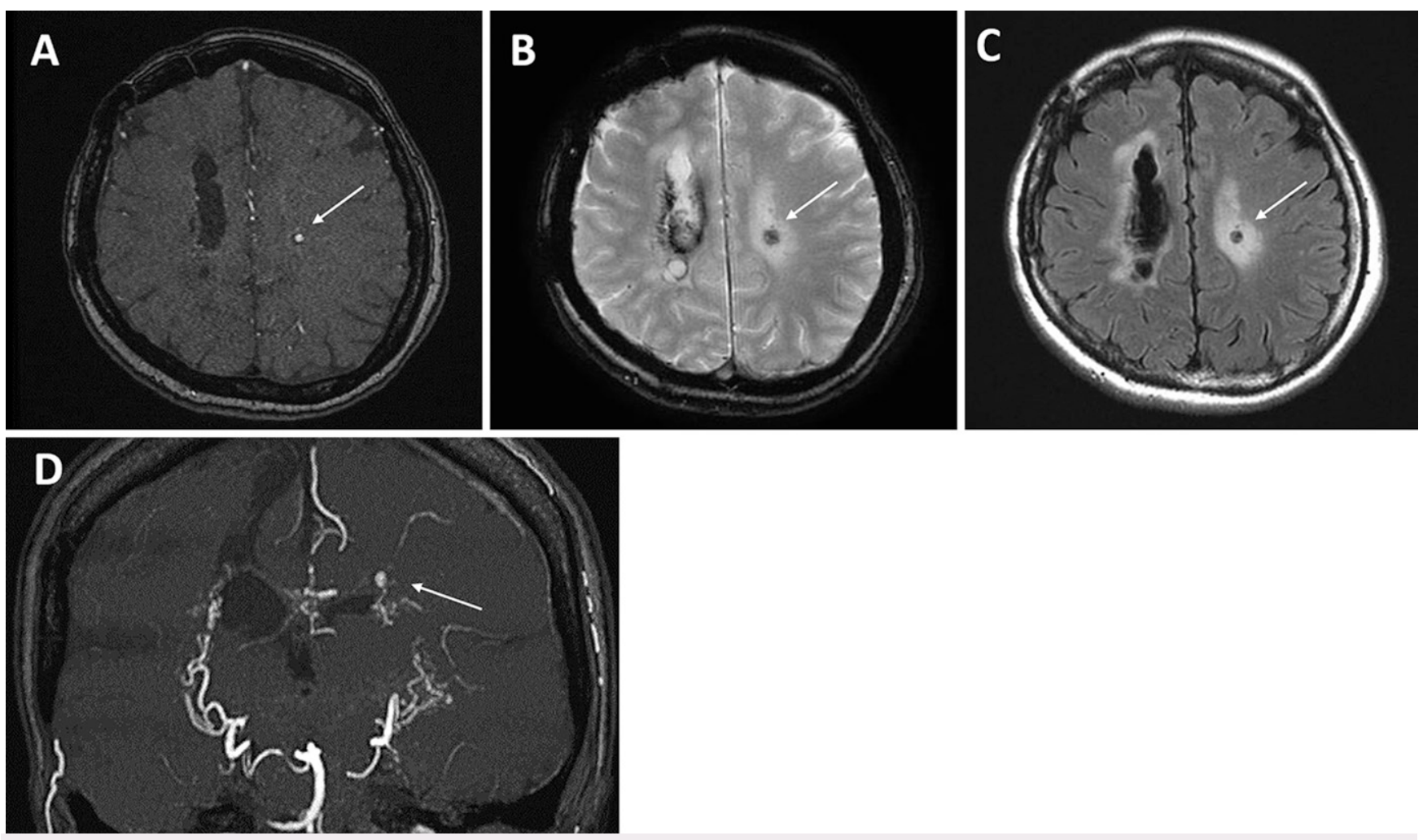

FIG. 1. MRI shows a small aneurysm formation in the left ventricle wall of the distal lateral choroidal artery (white arrows) that was suspected to be associated with minor bleeding. The aneurysm was developing on the preexisting choroidal anastomosis. A: Axial MRA image. B: T2-star image. C: Fluid-attenuated inversion recovery image. D: Coronal MRA image. 

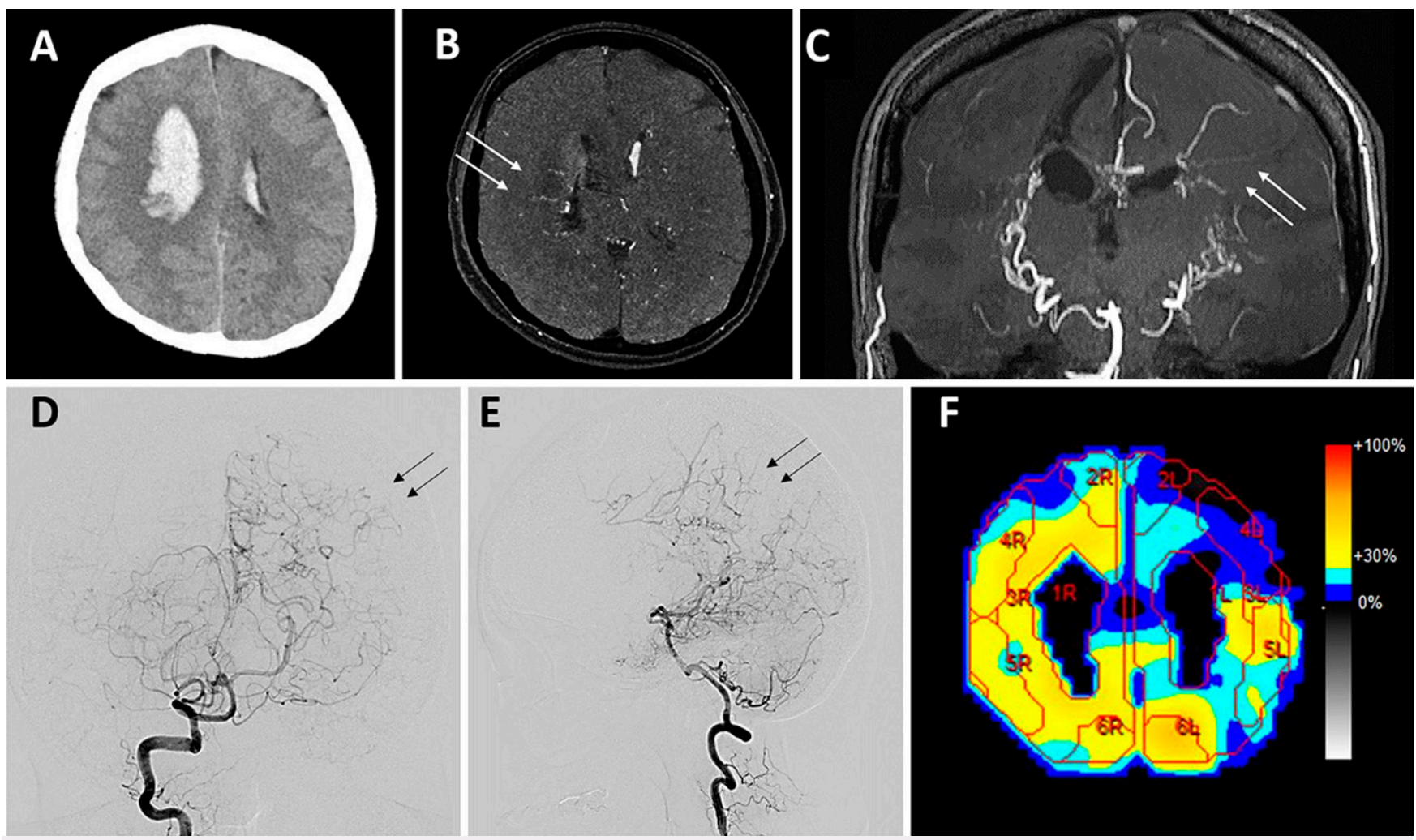

FIG. 2. Initial onset of hemorrhage in the right cerebral hemisphere had occurred 3 years earlier in case 1. A: CT image shows intracerebral hemorrhage in the right hemisphere and IVH. B: Choroidal anastomosis (white arrows) was suspected in the right hemorrhagic hemisphere on the axial image of the MRA. C: Development of a choroidal anastomosis in the left nonhemorrhagic hemisphere was detected on MRA imaging (coronal image; white arrows). D and E: Anterior-posterior and lateral views of left vertebral artery angiography show the development of a choroidal anastomosis (black arrows).

F: Single-photon emission CT scan demonstrates impaired CVR in the left nonhemorrhagic hemisphere.

severe right IVH and subarachnoid hemorrhage, and CT angiography (CTA) and MRA demonstrated the formation of a small aneurysm on the right ventricular wall (Fig. 4D-F).

She presented with a transient ischemic attack (TIA) associated with left-sided weakness at the age of 40 years. Conservative treatment was administered continuously thereafter; however, the patient developed a cerebral infarction of the left frontal lobe at the age of 69 years (Fig. 4A). Cerebral angiography demonstrated bilateral internal carotid artery stenosis (Suzuki stage III bilaterally), and the diagnosis of MMD was made. A dilated right posterior choroidal artery was also detected on the cerebral angiography with choroidal-type periventricular anastomosis (Fig. 4B and C). She did not undergo surgical intervention because of her advanced age, and conservative treatment was continued with $100 \mathrm{mg}$ of aspirin per day.

The development of a small aneurysm in the right posterior choroidal artery, indicating the etiology of the hemorrhage, was confirmed on DSA (Fig. 4G-I), and an increased choroidal anastomosis was also observed. Conservative treatment was continued because of the high risk of surgical intervention given the inaccessible aneurysm location and the patient's advanced age. She was transferred to another hospital for rehabilitation. However, almost 1 month after the initial bleeding episode, the hemorrhage recurred and she was admitted to our hospital again (Fig. 5A). The choroidal aneurysm was still observed on repeated CTA (Fig. 5B). Endovascular treatment was considered to prevent rebleeding from the ruptured aneurysm, and DSA was performed 3 days later, but the small aneurysm was not detected (Fig. 5C and D). The aneurysm was considered to have thrombosed and spontaneously regressed. Conservative treatment was continued, and she was transferred to the rehabilitation hospital again. No stroke event has occurred in the 7 years since her discharge from our hospital. MRI demonstrated no recurrence of the choroidal artery aneurysm, and the choroidal collaterals featured less development than those observed 7 years prior (Fig. 5E-G).

\section{Discussion}

Since the JAM Trial, periventricular anastomoses have been garnering increasing focus because they increase the risk of rebleeding in patients with hemorrhagic MMD, and the subanalysis of the JAM Trial indicates that periventricular anastomoses are associated with a potential risk of bleeding even in patients who are asymptomatic for MMD. ${ }^{7}$ Distal choroidal artery aneurysms are typically observed on the ventricular wall, which can cause IVH if they rupture in patients with MMD. Moreover, the resulting aneurysm is considered a pseudoaneurysm. ${ }^{13}$ Previous studies have suggested that local hemodynamic stress initiates aneurysm formation. ${ }^{11,14}$ Given the associated fragility and exposure to abnormal hemodynamics, the rupture risk of such aneurysms is relatively high. ${ }^{15,16}$ However, the existing literature is limited concerning descriptions about relevant clinical 

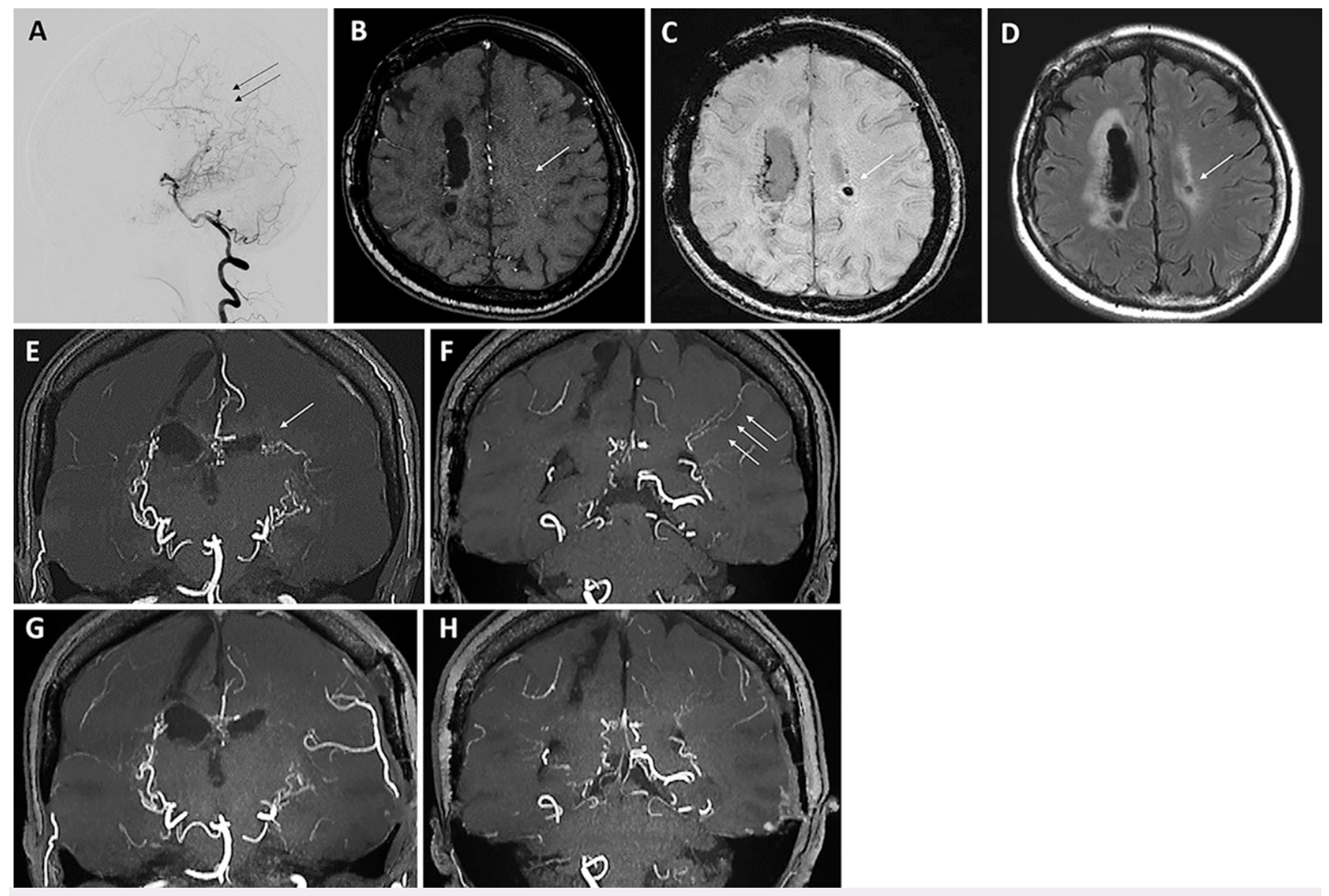

FIG. 3. A: Lateral views of right vertebral artery angiography (52 days after minor bleeding was detected) showing choroidal anastomosis (black arrows) with no obvious aneurysm in the left lateral choroidal artery. MRI demonstrated a thrombosed aneurysm (single white arrows). B: Axial MRA image. C: Susceptibility-weighted image (SWI). D: Fluid-attenuated inversion recovery image. E: Coronal MRA image. F: Choroidal anastomosis was still visible on the other site in the coronal MRA image (white arrows). $\mathbf{G}$ and $\mathbf{H}$ : The coronal MRA images after bypass surgery demonstrated the regression of choroidal anastomosis.

courses in patients with choroidal artery aneurysms and prognoses after aneurysm rupture, ${ }^{17}$ the natural course of choroidal artery aneurysms, including aneurysm development, in choroidal collaterals has rarely been reported. In our patients, both choroidal artery aneurysms developed on preexisting choroidal collaterals during the follow-up period and eventually ruptured. Funaki et al. reported in the subanalysis of the JAM Trial that the risk of de novo hemorrhage in the nonhemorrhagic hemispheres was significantly higher in patients positive for choroidal anastomosis than in patients negative for choroidal anastomosis, and the surgical group positive for choroidal anastomosis had no hemorrhagic event during the 5-year follow-up period. ${ }^{7}$ In case 1 , a choroidal anastomosis was detected in the nonhemorrhagic hemisphere of the surgical patient. The peripheral aneurysm developed 2 years after the surgery, with an asymptomatic minor hemorrhage in a mirror image of the previous contralateral hemorrhage. These findings indicate that careful observation of surgical patients is required even in the presence of a contralateral choroidal anastomosis.

The second case of an older patient with ischemic MMD was particularly unusual because she presented with a rare hemorrhagic transformation in the contralateral hemisphere long after the ischemic event. Ischemic episodes are the most common symptom among older patients with MMD; hemorrhagic MMD is less prevalent than ischemic MMD. ${ }^{18}$ Hemorrhagic events in older patients with MMD are associated with high rates of morbidity and mortality; in particular, rebleeding is a significant risk factor for poor outcomes. ${ }^{19}$ In our case, a choroidal anastomosis was detected 6 years before a hemorrhage resulting from a lateral posterior choroidal artery aneurysm. Hori et al. reported that the increasing age in the Japanese population is associated with dilation and extension of the posterior choroidal artery, which is associated with the potential risk of hemorrhagic events. $^{20}$ To date, our patient is the oldest patient with MMD in whom a choroidal artery aneurysm has been reported to develop and rupture.

Interestingly, in both cases, the aneurysm spontaneously regressed after bleeding. Some studies have reported spontaneous regression of distal choroidal artery aneurysms with regressed choroidal anastomosis after 6 weeks to 11 months..$^{10,21,22}$ In case 1, the regression occurred almost 2 months after minor bleeding, whereas it occurred only 3 days after rebleeding in case 2 . In both cases, choroidal 

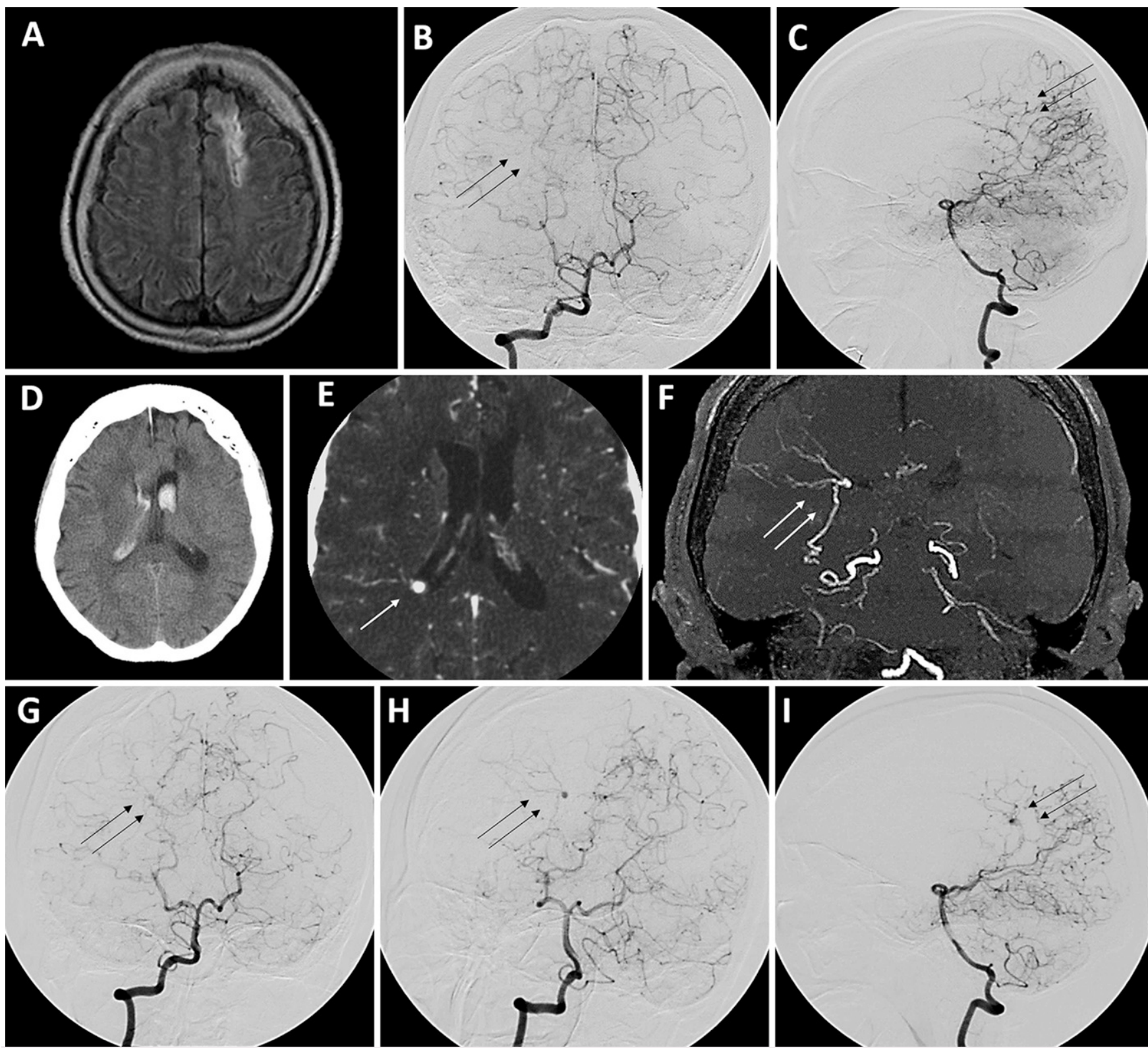

FIG. 4. Onset of ischemic stroke had occurred 6 years earlier in case 2. A: Cerebral infarction occurred in the left frontal lobe. B and C: Anteriorposterior and lateral views of the right vertebral artery angiography. Development of choroidal collaterals was observed (black arrows). D: CT imaging shows IVH. E: CTA imaging detected a small aneurysm in the distal lateral posterior choroidal artery of the right lateral ventricle wall (white arrow). F: MRA image shows a distal lateral posterior choroidal artery aneurysm arising from choroidal anastomosis (white arrows). G-I: Anterior-posterior, left anterior oblique, and lateral views of the right vertebral artery angiography demonstrate a distal choroidal artery aneurysm in the right lateral posterior choroidal artery with the development of a choroidal anastomosis (black arrows).

anastomosis was still observed after spontaneous regression. Bypass surgery was performed in case 1, after which the choroidal anastomosis regressed, and no rebleeding occurred. Bypass surgery is considered effective for stabilizing hemodynamic stress on choroidal anastomosis and preventing aneurysm formation. ${ }^{11,23}$ In the meantime, the spontaneous regression of an aneurysm is possible; however, some authors have reported a high risk of associated rebleeding. ${ }^{24}$ Notably, in case 2, the aneurysm caused the rebleeding within 1 month, although it thrombosed within 3 days after the rebleed. To the best of our knowledge, the spontaneous thrombosis of a choroidal artery aneurysm after rebleeding has not been described previously, and the 3-day period is the shortest in spontaneous regression of ruptured choroidal artery aneurysms.

In the presence of fragile periventricular anastomoses, the development of peripheral artery aneurysms appears to be associated with hemodynamic stress instability in the collateral vessels. In particular, the presence of a choroidal anastomosis is thought to be predictive of unstable hemodynamic conditions and a high risk 

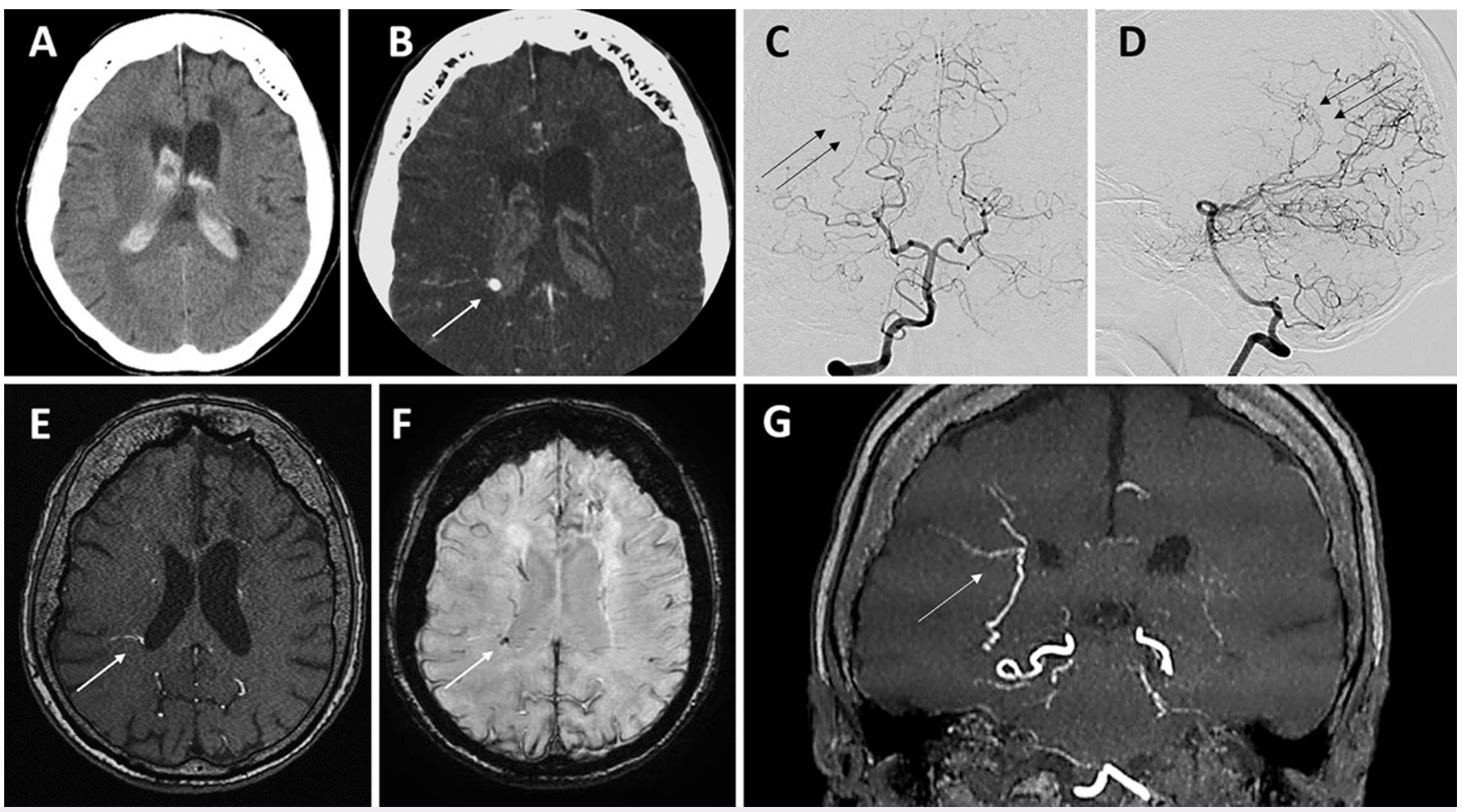

FIG. 5. A: CT imaging shows rebleeding. B: A distal right lateral choroidal artery aneurysm was the origin of the hemorrhage (white arrow). Anteriorposterior (C) and lateral (D) views of the right vertebral artery angiography demonstrate no obvious aneurysm on choroidal anastomosis (black arrows); it was suspected that the aneurysm had spontaneously regressed. The MRI performed 7 years after rebleeding showed less development of choroidal anastomosis, and no recurrence of the aneurysm was observed (single white arrows, E-G). E: Axial MRA image. F: SWI. G: Coronal MRA image.

of hemorrhagic events. ${ }^{14}$ Wang et al. reported lateral posterior choroidal collateral anastomosis to be a predictive marker of recurrent ipsilateral hemorrhage. ${ }^{12}$ Conversely, the emergence of microaneurysms on choroidal collateral vessels is rarely reported, and Matsushige et al. reported that ventricular microaneurysms in the posterior choroidal collateral vessels of patients with MMD can be detected by 7-T time-of-flight MRA. ${ }^{25}$ This finding indicates that such fragile choroidal collaterals are associated with the potential risk of aneurysm formation.

Our cases indicate that the development of choroidal anastomosis, especially in patients with preceding stroke events, may potentially be accompanied by peripheral aneurysm formation and rupture risk in the nonsurgical hemisphere. One patient had a history of hemorrhage in the contralateral hemisphere, and the other experienced an ischemic stroke event in the contralateral hemisphere. Furthermore, the hemodynamic states in the hemispheres with a newly developed aneurysm of both patients seemed unstable. The impairment of CVR was observed in case 1, and the patient in case 2 presented with a history of TIA. These clinical findings indicate that the unstable hemodynamic state without surgical intervention triggered the development of choroidal anastomosis, causing a peripheral aneurysm and eventual rupture. These aneurysms are thought to be pseudoaneurysms, and bypass surgery is effective for stabilizing abnormal hemodynamic stress and consequently preventing further bleeding while promoting gradual aneurysm thrombosis. ${ }^{23}$ On the contrary, endovascular surgery is effective in terms of prompt and radical treatment of aneurysms based on direct embolization if catheter access is feasible. ${ }^{13,26}$ Bypass surgery was performed in case 1, and choroidal anastomosis remarkably regressed only 2 weeks after surgery; however, in case 2, the older patient with MMD was not treated surgically because of her advanced age. The patient experienced no stroke event and no recurrence of the aneurysm for more than 7 years after the episode of rebleeding, although careful observation is required in patients with MMD who have choroidal anastomosis. This is an extremely rare case that demonstrates the long-term prognosis of a choroidal anastomosis in nonsurgical patients with MMD after rebleeding.

\section{Observations}

The natural course comprising the development, rupture, and spontaneous thrombosing of choroidal artery aneurysms in preexisting choroidal anastomoses was described in 2 patients with MMD. Both patients had a previous stroke event in the contralateral hemisphere; one was hemorrhagic and the other ischemic. Asymptomatic minor bleeding occurred in the contralateral hemisphere of a previous hemorrhagic episode in 1 patient. Hemorrhagic transformation occurred in the long-term follow-up of the elderly patient. In 1 patient, spontaneous regression of the aneurysm occurred within 2 months after minor bleeding, whereas it occurred within 3 days after rebleeding in the other patient. Development of choroidal artery aneurysms has rarely been reported. This report also documents the follow-up of thrombosed peripheral aneurysms with choroidal collaterals after bleeding in 2 patients with MMD who each had a previous stroke event. Neither de novo aneurysm formation nor additional hemorrhage was observed in either patient in the subsequent follow-up. 


\section{Lessons}

The formation of distal choroidal artery aneurysms may potentially be related to hemodynamic stress associated with the development of choroidal anastomoses. Patients with MMD and fragile collaterals in the nonsurgical hemisphere with a preceding stroke event and impaired cerebral blood flow require careful follow-up, including elderly patients. Bypass surgery could be considered to stabilize the hemodynamic stress; however, spontaneous regression of aneurysms can occur and remain over the longer term.

\section{References}

1. Kuroda S, Houkin K. Moyamoya disease: current concepts and future perspectives. Lancet Neurol. 2008;7(11):1056-1066.

2. Kobayashi E, Saeki N, Oishi H, et al. Long-term natural history of hemorrhagic moyamoya disease in 42 patients. J Neurosurg. 2000; 93(6):976-980.

3. Miyamoto S, Yoshimoto T, Hashimoto N, et al. Effects of extracranial-intracranial bypass for patients with hemorrhagic moyamoya disease: results of the Japan Adult Moyamoya Trial. Stroke. 2014;45(5):1415-1421.

4. Funaki T, Takahashi JC, Houkin K, et al. High rebleeding risk associated with choroidal collateral vessels in hemorrhagic moyamoya disease: analysis of a nonsurgical cohort in the Japan Adult Moyamoya Trial. J Neurosurg. 2019;130(2):525-530.

5. Fujimura M, Funaki T, Houkin K, et al. Intrinsic development of choroidal and thalamic collaterals in hemorrhagic-onset moyamoya disease: case-control study of the Japan Adult Moyamoya Trial. J Neurosurg. 2019;130(5):1453-1459.

6. Funaki T, Takahashi JC, Houkin K, et al. Angiographic features of hemorrhagic moyamoya disease with high recurrence risk: a supplementary analysis of the Japan Adult Moyamoya Trial. J Neurosurg. 2018;128(3):777-784.

7. Funaki T, Takahashi JC, Houkin K, et al. Effect of choroidal collateral vessels on de novo hemorrhage in moyamoya disease: analysis of nonhemorrhagic hemispheres in the Japan Adult Moyamoya Trial. J Neurosurg. 2019;132(2):408-414.

8. Kawaguchi S, Sakaki T, Morimoto T, et al. Characteristics of intracranial aneurysms associated with moyamoya disease. A review of 111 cases. Acta Neurochir (Wien). 1996;138(11): 1287-1294.

9. Iwama T, Todaka T, Hashimoto N. Direct surgery for major artery aneurysm associated with moyamoya disease. Clin Neurol Neurosurg. 1997;99(suppl 2):S191-S193.

10. Kodama N, Suzuki J. Moyamoya disease associated with aneurysm. J Neurosurg. 1978;48(4):565-569.

11. Kanamori F, Takasu S, Ota S, Seki Y. Prevention of the rerupture of collateral artery aneurysms on the ventricular wall by early surgical revascularization in moyamoya disease: report of two cases and review of the literature. World Neurosurg. 2018;109:393-397.

12. Wang J, Yang Y, Li X, et al. Lateral posterior choroidal collateral anastomosis predicts recurrent ipsilateral hemorrhage in adult patients with moyamoya disease. AJNR Am J Neuroradiol. 2019; 40(10):1665-1671.

13. Kim SH, Kwon OK, Jung CK, et al. Endovascular treatment of ruptured aneurysms or pseudoaneurysms on the collateral vessels in patients with moyamoya disease. Neurosurgery. 2009;65(5): 1000-1004.

14. Morioka M, Hamada J, Kawano T, et al. Angiographic dilatation and branch extension of the anterior choroidal and posterior communicating arteries are predictors of hemorrhage in adult moyamoya patients. Stroke. 2003;34(1):90-95.

15. Takahashi JC, Miyamoto S. Moyamoya disease: recent progress and outlook. Neurol Med Chir (Tokyo). 2010;50(9):824-832.
16. Adams HP Jr, Kassell NF, Wisoff HS, Drake CG. Intracranial saccular aneurysm and moyamoya disease. Stroke. 1979;10(2): 174-179.

17. Rhim JK, Cho YD, Jeon JP, et al. Ruptured aneurysms of collateral vessels in adult onset moyamoya disease with hemorrhagic presentation. Clin Neuroradiol. 2018;28(2):191-199.

18. Ge P, Zhang $Q$, Ye X, et al. Clinical features, surgical treatment, and long-term outcome in elderly patients with moyamoya disease. World Neurosurg. 2017;100:459-466.

19. Gupta R, Moore JM, Adeeb N, et al. Clinical presentation, progression, and treatment outcomes of moyamoya disease in the elderly. Acta Neurochir (Wien). 2016;158(12):2409-2414.

20. Hori S, Kashiwazaki D, Yamamoto S, et al. Impact of interethnic difference of collateral angioarchitectures on prevalence of hemorrhagic stroke in moyamoya disease. Neurosurgery. 2019; 85(1):134-146.

21. Miyake $H$, Ohta T, Kajimoto $Y$, et al. Intraventricular aneurysmsthree case reports. Neurol Med Chir (Tokyo). 2000;40(1):55-60.

22. Yamada H, Saga I, Kojima A, Horiguchi T. Short-term spontaneous resolution of ruptured peripheral aneurysm in moyamoya disease. World Neurosurg. 2019;126:247-251.

23. Kuroda S, Houkin K, Kamiyama H, Abe H. Effects of surgical revascularization on peripheral artery aneurysms in moyamoya disease: report of three cases. Neurosurgery. 2001;49(2):463-468.

24. Yoshida Y, Yoshimoto T, Shirane R, Sakurai Y. Clinical course, surgical management, and long-term outcome of moyamoya patients with rebleeding after an episode of intracerebral hemorrhage: an extensive follow-up study. Stroke. 1999;30(11): 2272-2276.

25. Matsushige T, Kraemer M, Schlamann M, et al. Ventricular microaneurysms in moyamoya angiopathy visualized with $7 \mathrm{~T}$ MR angiography. AJNR Am J Neuroradiol. 2016;37(9):1669-1672.

26. Ni W, Jiang $H, X u B$, et al. Treatment of aneurysms in patients with moyamoya disease: a 10-year single-center experience. $J$ Neurosurg. 2018;128(6):1813-1822.

\section{Disclosures}

The authors report no conflict of interest concerning the materials or methods used in this study or the findings specified in this paper.

\section{Author Contributions}

Conception and design: Suzuki, Hasegawa, Okamoto, Saito, Fujii. Acquisition of data: Suzuki, Ando. Analysis and interpretation of data: Suzuki, Okamoto, Saito. Drafting the article: Suzuki, Hasegawa, Okamoto, Fujii. Critically revising the article: Suzuki, Okamoto, Shibuya, Takahashi. Reviewed submitted version of manuscript: Suzuki, Hasegawa, Okamoto, Ando, Shibuya, Takahashi, Saito, Fujii. Approved the final version of the manuscript on behalf of all authors: Suzuki. Administrative/technical/material support: Suzuki, Hasegawa. Study supervision: Suzuki, Okamoto, Shibuya, Takahashi, Oishi, Fujii.

\section{Correspondence}

Tomoaki Suzuki: Brain Research Institute, Niigata University, Niigata, Japan. t.suzuki2078@gmail.com. 